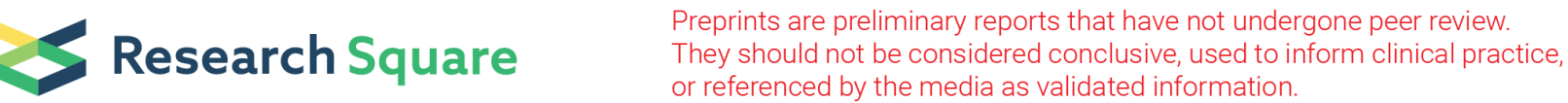

\section{The Absence of 4' Methyl on the D-ring of Strigolactone does not Prevent Inhibits of Tillering in Rice Through Activation of the Strigolactone Signaling Pathway}

Yuexin Liu

Nanjing Agricultural University

Chenyu Rong

Nanjing Agricultural University

Shi Wang

Nanjing Agricultural University

Lei Wu

Nanjing Agricultural University

Yanfeng Ding

Nanjing Agricultural University

Chengqiang Ding ( $\nabla$ dingcq@njau.edu.cn )

Nanjing Agricultural University https://orcid.org/0000-0001-6061-1979

\section{Research Article}

Keywords: D10, D14, rice, shoot branching, strigolactone analog, tiller

Posted Date: February 2nd, 2022

DOI: https://doi.org/10.21203/rs.3.rs-1244555/v1

License: (c) (i) This work is licensed under a Creative Commons Attribution 4.0 International License.

Read Full License 


\section{Abstract}

Strigolactones (SLs) are a new class of carotenoid-derived phytohormones that are essential for developmental processes that shape plant architecture. One consistent feature of natural and synthetic compounds with SL-like activity is the presence of an enol-ether-connected methyl butenolide moiety (Dring). Here, we synthesized a suite of special chemicals without methyl in the butenolide moiety that exhibited the same biological activity as SLs in rice (Oryza sativa), including shoot branching suppression. D10 and D14 are crucial genes involved in SL synthesis and signaling pathways, respectively. We found that 15 of the 29 newly-synthesized chemicals possessed SL-like activity against the rice $d 10$ mutant and were invalid for the $d 14$ mutant. We also discovered that the SL receptor D14 could physically interact with d53 in the presence of these chemicals, indicating that their molecular structures matched the ligand perception site pocket of D14. Moreover, examining the transcript levels of SL downstream response genes after treatment with each compound revealed how they operated in the SL pathway and guided other potential signaling pathways. In combination with these compounds' structures, our results suggest that SL signaling does not require a methyl substituent in the D-ring. Our combined genetic and biochemical data reveal the structure-activity relationships of SLs and their potential for shaping plant architecture.

\section{Introduction}

Strigolactones (SLs) are a group of carotenoid-derived phytohormones that were first identified as seed germination stimulants of root parasitic plants, such as Striga and Orobanche (Cook et al. 1966). SLs have also been implicated in other processes, including the stimulation of hyphal branching in arbuscular mycorrhizal fungi (Akiyama and Hayashi 2006), the regulation of leaf morphology (Scaffidi et al. 2013) and root architecture (Koltai 2011), and the inhibition of bud outgrowth to reduce shoot branching (Gomez-Roldan et al. 2008). Studies into the effects of SLs have isolated a series of enhanced shoot branching, and have identified dwarfing mutants including ramosus $(\mathrm{rms})$ of pea, more axillary growth (max) of Arabidopsis, and dwarf (d) mutants of rice (Wang et al. 2018); SL biosynthesis and signal transduction were identified in these mutants. The primary step of SL biosynthesis involves the conversion of all-trans- $\beta$-carotene into 9-cis- $\beta$-carotene by the isomerase D27 in rice (Alder et al. 2012). Sequential actions of carotinoid cleavage deoxygenase 7 (CCD7) and CCD8, as encoded by DWARF17 (D17) and D10, lead to the formation of carlactone, which is the precursor for SLs (Arite et al. 2007; Umehara et al. 2008). After several further types of catalysis, carlactone is converted to SLs (Al-Babili and Bouwmeester 2015; Zhang et al. 2014). The SL receptor DWARF14 (D14) was initially established from an SL-insensitive rice mutant, $d 14$, which showed dwarf phenotypes and increased shoot branching (Arite et al. 2009). As an $\alpha / \beta$-fold hydrolase, D14 is also responsible for both the perception and deactivation of bioactive SLs (Seto et al. 2019). In rice, the complex for the perception of SLs also includes the negative regulators D53 and suppressor of max 21 -like (SMXL) 6/7/8 in Arabidopsis and the F-box proteins D3 and more axillary growth 2 (MAX2) (Jiang et al. 2013; Mach 2015; Wang et al. 2015; Zhou et al. 2013). SL-responsive genes begin to play their roles after the ubiquitination and subsequent degradation of D53 
through the 26s proteasome pathway. In addition to the feedback regulation of $D 53$ and the inhibitor of bud outgrowth BRANCHED1 (BRC1) (Dun et al. 2012), cytokinin oxidase/dehydrogenase 9 (OsCKX9) and the type-A response regulator OSRR 5 have been identified as the primary and secondary SL-responsive genes, respectively (Duan et al. 2019).

To date, several artificial SL analogs have been chemically synthesized based on the structures of natural SLs identified from root exudates; rac-GR24 is the most widely used SL analog. SLs have been identified as a member of the terpenoids, which consist of a tricyclic lactone (ABC ring) and a hydroxymethyl butanolide (D-ring) that are connected via an enol ether bond. Studies into SL bioactivity regarding the induction of root parasitic seed germination have revealed that the C-D-ring may be important for biological activity and that modifying the D-ring reduces SL bioactivity (Mangnus et al. 1992; Mangnus and Zwanenburg 1992). Furthermore, 5-(4-chlorophenoxy)-3-methylfuran-2(5H)-one and several of its derivatives have also been found to possess SL-like activity inhibit shoot branching in rice d10-1 mutants (Fukui et al. 2011a). In garden peas, it has been demonstrated that the presence of a Michael acceptor and a methylbutenolide or dimethylbutenolide motif in the same molecule is essential for controlling plant architecture (Boyer et al. 2012). The structure-activity relationships of SLs have been extensively studied regarding inducing the seed germination of root parasitic plants and inhibiting shoot branching (Xie 2016; Zwanenburg et al. 2009). Furthermore, apparent SL biological activity has been greatly influenced by differences in plant species and application methods (Umehara et al. 2015). The stereoisomers of SLs are also critical for hormonal activity (Scaffidi et al. 2014; Umehara et al. 2015).

Recently, SL structural requirements have been further conjectured because new SL signal transductions have been found. Previously, SL has been hydrolyzed into a covalently-linked intermediate molecule (CLIM), which is closely associated with the conformational change of AtD14 (Yao et al. 2016). When the hydrolase receptor D14 receives intact SL molecules, the D14 active signaling state is triggered and SL is hydrolyzed into a CLIM (Seto et al. 2019). Therefore, the intact SL structure, including the ABC ring, is potentially significant for biological activity. Karrikins are also butenolide compounds similar to SLs, and the receptor for karrikins (KARRIKIN INSENSITIVE 2; KAI2) is a homologous protein of D14. Therefore, KAI2-dependent signalling and structure of Karrikins become a hot topic in research. The butenolide methyl group is considered nonessential to KAI2 signalling, and Desmethyl-GR24 is active by KAI2 but not D14 (Yao et al. 2021). However, to date, the necessity of the methyl substituent in the D-ring for bioactivity has not been investigated regarding the inhibition of tillering through SL signalling in rice.

Here, therefore, we synthesized 29 analogs of SL that did not possess methyl groups and evaluated their effects on shoot branching inhibition. To determine whether these compounds acted through the SL pathway, we selected several to treat the SL signaling mutant $d 14$ and investigated whether they could inhibit the extreme tillering of $d 14$. Through these investigations, we aimed to explore the structural requirements of SLs for shoot branching inhibition in rice and provide new insights into SL signaling pathways.

\section{Materials And Methods}




\section{Chemicals}

The first step comprised bromination at the furan-2(5H)-one using $\mathrm{N}$-bromosuccinimide, which was then coupled with a variety of materials under basic conditions to obtain a crude mixture of compounds (Fig. 1a).

Preparation of phenolic compounds: To a mixture of $\mathrm{K}_{2} \mathrm{CO}_{3}(2.5 \mathrm{mmol}, 1.25$ equiv. $)$ and TBAB $(0.2 \mathrm{mmol}$, 0.1 equiv.) in $\mathrm{H}_{2} \mathrm{O}(10 \mathrm{~mL}$ ), phenols ( 2 mmol, 1.0 equiv.) dissolved in dichloromethane $(5 \mathrm{~mL})$ was added dropwise, the mixture was stirred at room temperature for $10 \mathrm{~min}, 5$-bromofuran-2(5H)-one $(2 \mathrm{mmol}, 1.0$ equiv.) dissolved in dichloromethane $(5 \mathrm{~mL})$ was added dropwise, and the resulting mixture was stirred for $8 \mathrm{~h}$ at room temperature. The mixture was extracted with dichloromethane $(3 \times 5 \mathrm{~mL})$. The combined organic phase was dried over $\mathrm{Na}_{2} \mathrm{SO}_{4}$ and concentrated in vacuo. The crude mixtures were purified by flash chromatography to afford pure products.

Preparation of carboxylic acid compounds/ester compounds/amino acid-derived compound: To a mixture of $\mathrm{CsCO}_{3}$ (3 mmol, 1.5 equiv.), benzoic acid ( $2 \mathrm{mmol}, 1.0$ equiv.) in $\mathrm{N}, \mathrm{N}$-Dimethylformamide (1 $\mathrm{mL}$ ) was added, the mixture was stirred at room temperature for $10 \mathrm{~min}, 5$-bromofuran-2(5H)-one (2 mmol, 1.0 equiv.) dissolved in $\mathrm{N}, \mathrm{N}$-Dimethylformamide $(2 \mathrm{~mL})$ was added dropwise and the resulting mixture was stirred for $4 \mathrm{~h}$ at room temperature. The mixture was extracted with EtOAc $(3 \times 5 \mathrm{~mL})$. The combined organic phase was dried over $\mathrm{Na}_{2} \mathrm{SO}_{4}$ and concentrated in vacuo. The crude mixtures were purified using flash chromatography to afford pure products. All chemical drugs and reagents in this experiment were purchased from Shanghai Energy Chemical Co., Ltd.

\section{Plant Materials}

We used the Oryza sativa japonica cultivar (cv.) Nipponbare to perform compound gradient tests and leaf spraying tests, as well as to determine the effects of compounds on gene transcription levels. The tillering dwarf mutants, d10-1and d14-1 in the Shiokari background were used to test the effectiveness of the compounds. (Arite et al. 2007; Arite et al. 2009).

\section{Hydroponic culture of Rice for the Evaluation of Branching Inhibitory Activity}

Rice seeds were soaked, pre-germinated at $28^{\circ} \mathrm{C}$ in the dark for $72 \mathrm{~h}$, and then sown in the soil. After 14 days, Seedlings were transferred onto boards floating on rectangular plastic trays $(23 \times 14 \times 9 \mathrm{~cm})$ filled with $3 \mathrm{~L}$ of complete-strength, modified Yoshida nutrient solution containing $2.0 \mathrm{mM} \mathrm{NH}_{4} \mathrm{NO}_{3}, 0.32 \mathrm{mM}$ $\mathrm{KH}_{2} \mathrm{PO}_{4} \cdot 2 \mathrm{H}_{2} \mathrm{O}, 0.3 \mathrm{mM} \mathrm{MgCl} \cdot 6 \mathrm{H}_{2} \mathrm{O}, 11.0 \mu \mathrm{M} \mathrm{MnCl}_{2} \cdot 4 \mathrm{H}_{2} \mathrm{O}, 18.5 \mu \mathrm{M} \mathrm{H}_{3} \mathrm{BO}_{3}, 0.6 \mu \mathrm{M} \mathrm{Na}_{2} \mathrm{MoO}_{4} \cdot 2 \mathrm{H}_{2} \mathrm{O}, 1.4 \mu \mathrm{M}$ $\mathrm{ZnSO}_{4} \cdot 7 \mathrm{H}_{2} \mathrm{O}, 1.6 \mu \mathrm{M} \mathrm{CuSO}_{4} \cdot 5 \mathrm{H}_{2} \mathrm{O}, 0.66 \mathrm{mM} \mathrm{CaCl}_{2}, 50.0 \mu \mathrm{M} \mathrm{FeSO}_{4} \cdot 7 \mathrm{H}_{2} \mathrm{O}$, and $50.0 \mu \mathrm{M} \mathrm{Na}{ }_{2} \mathrm{EDTA}_{2} \cdot 2 \mathrm{H}_{2} \mathrm{O}$. The nutrient solution was refreshed every four days to maintain a pH of 5.5. Treatment commenced 7 days after transplantation by supplementing the compound. Each test chemical was dissolved in dimethyl sulfoxide (DMSO) and added to the hydroponic culture solution. The final concentration of the solution was adjusted to $50.0 \mu \mathrm{M}$. 


\section{Y2H assay}

The coding region of rice $d 53$ was synthesized and sequenced via Sanger sequencing (Genewiz,

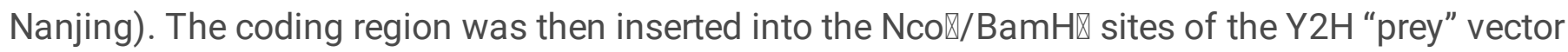
pGADT7 (Clontech, USA). The coding region of rice D14 was amplified using polymerase chain reaction (PCR). The gene-specific primers used for PCR were 5'-CATATGCTGCGATCGACGCATCCGCCGCCCAG-3' and 5'-GAATTCTTAGTACCGGGCGAGAGCGCGG-3' (Genewiz). The resulting amplicon was inserted into the pEASY-Blunt Zero vector (TransGen Biotech, Beijing). The vector was identified using Sanger

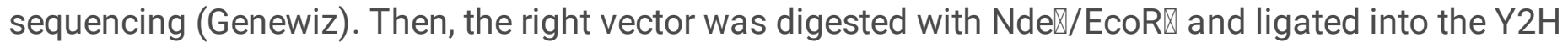
"bait" vector pGBKT7 (Clontech). The constructs were co-transformed into the yeast (Saccharomyces cerevisiae) strain Y2HGold (Clontech) and the resultant yeast strains were grown on SD-Leu/-Trp plates for $3 \mathrm{~d}$ at $30^{\circ} \mathrm{C}$. Interactions between two proteins were examined on the control (LT [SD-Leu/-Trp]) and selective media (LTHA [SD-Leu/-Trp/-His/-Ade]) containing $5 \mu \mathrm{M} \mathrm{GR24} \mathrm{or} 50 \mu \mathrm{M}$ of each compound from ethanol-dissolved stock solution (ethanol was used as a control). The plates were incubated for 10 days at $30^{\circ} \mathrm{C}$. Yeast strains containing d53 or D14 in combination with pGADT7 or pGBKT7 were used as controls.

\section{RNA extraction and cDNA synthesis}

Total RNA was extracted from frozen samples using the EZNATM Plant RNA Kit (Omega Bio-tek, USA) and treated with RNase-free DNase I (Omega) to remove any remaining genomic DNA. First-strand cDNA was synthesized using 500 ng total RNA with the PrimeScript RT Reagent Kit (TaKaRa, Dalian, China), following the manufacturer's protocol.

\section{Quantitative real-time reverse transcriptase (RT)-PCR}

Quantitative RT-PCR (20 $\mu \mathrm{L}$ reaction volume) was performed using $2 \mu \mathrm{l}$ CDNA, $1 \mu \mathrm{M}$ of each gene-specific primer, and SYBR ${ }^{\circledR}$ Premix Ex Taq ${ }^{\text {TM }}$ Kit (TaKaRa) in an Applied Biosystems 7300 Sequence Detection system (Life Technologies Ltd, Carlsbad, CA, USA), according to the manufacturer's instructions. The reaction was performed using the default conditions of the ABI 7300 SDS Software 1.3 .1 as follows: $90^{\circ} \mathrm{C}$ for $30 \mathrm{~s}$, followed by 40 cycles of $5 \mathrm{~s}$ at $95^{\circ} \mathrm{C}$ and $30 \mathrm{~s}$ at $60^{\circ} \mathrm{C}$. The data were analyzed using the relative quantification method. The values presented herein are the means of three biological replicates per treatment, each of which were prepared in three technical replicates. Error bars indicate standard deviation from the mean. The rice ACTIN was used as an internal control. The gene-specific primers used for qRT-PCR were as follows:

ACTIN (5'-CAATCGTGAGAAGATGACCC-3' and 5'-GCTACGAGCTTCCTGATGGAC-3'); D53 (5'-

GAGGAGGATAGGAAACCTGTGCC-3' and 5'-GTACCAGCAGTGAAAGGAGAC-3'); OsCKX9 (5'-

CTATCCTCAGCACTTGGCCC-3' and 5'-CAGGAGTGGCAGTCCCATTT-3'); OsTB1 (5'-

CCTACCATGAGAGAAGAGACCA-3' and 5'-TCAACAGGTGCTTGGAGGAA-3'); OsRR5 (5'-

TGGCGGATATGTGAAGGTGAA-3' and 5'-CTTGTCCATCCTCGGCTCATA-3') (Genewiz). 


\section{Results}

\section{Synthesis of SL Analogs and their Effects on Rice Plant Architecture}

As SLs can signal through D14 without binding to the D-ring methyl, we hypothesized that the D-ring methyl group is not necessary for SL signaling, and that the ABC ring is important for SL activity. To investigate these possibilities, we chemically synthesized 29 compounds using the modified procedures reported in the methods section of this study (Fig. 1a). Based on the type of material, we divided these compounds into four categories: phenols, carboxylic acids, esters, and amino acids (Fig. 1b-e).

We then evaluated the inhibitory activities of the newly-synthesized compounds on hydroponically grown $d 10$ mutants, as reported previously (Fukui et al. 2013). In this test, we used the SL-deficient $d 10$ mutant, in which a gene encoding the SL biosynthesis enzyme (CCD8) was mutated. This mutant showed more tillering; however, it reverted to showing less tillering after the application of SLs. We examined the inhibitory effects of all of the synthesized compounds in this assay system at $50 \mu \mathrm{M}$ (Fig. 2). All of the synthesized compounds listed in Figure 1 are 2(5H)-furanone derivatives; they showed different biological activities depending on the category of the compound. Phenolic compounds (i.e., compounds 1-14), were found to be widely active; the majority showed reductions in shoot branching, plant height, and root length. Carboxylic acid compounds (compounds 15-26) were partly active; a minority inhibited tillering numbers, but the majority also showed reductions in root length. Interestingly, the two ester compounds (compounds 27 and 28) had different effects on tillering inhibition; the sole amino acid compound was not very active. Combined with the results presented in Figure 2, this showed that the carboxylic acid compounds were likely less active than the phenolic compounds. This result obtained here by studying the structure-activity relationships of the derivatives could be useful for future research into the SL pathway.

\section{Effective Concentrations of Compounds for Inhibition of Tillering}

GR24, as a synthetic analog that can effectively inhibit tillering, can inhibit tillering at a concentration of as low as $2 \mu \mathrm{M}$. Here, the compound used in this study inhibited tillering at $50 \mathrm{Mm}$, but whether it would work at lower concentrations is unclear. We used Nipponbare, which is a widely used rice cultivar, for our tests. Experiments at five different concentrations revealed that compound 6 had an effect at $30 \mu \mathrm{M}$, and that its effect was more obvious at $50 \mu \mathrm{M}$ (Fig. 3a). It was difficult to conduct concentration gradient tests for each compound because the structure of each compound likely affected its effective concentration. However, the absence of 4 'methyl on the $D$ rings of the studied compounds appeared to make them less effective than GR24.

Here, we also experimented with the application of new synthetic analogs. In addition to adding each compound to the hydroponic solution used in the experiments, we also sprayed the leaves of the 
experimental crops and found that leaf treatment did not inhibit tillering (Fig. 3b). Their inability to treat leaves would likely limit the applied usage of the studied compounds, especially when attempting to inhibit tillering in field-grown crops.

\section{Effects of Chemicals on the Tillering of d10 and d14 Mutants}

To confirm the activities of the studied compounds regarding branching inhibition and discriminate these from possible toxicity, each compound was tested on an SL response mutant (d14). In the case of toxicity, branching inhibition was observed in this mutant. However, if the compound being tested was active using the $d 10 \mathrm{SL}$-deficient mutant, then said compound should not have inhibited bud outgrowth at $d 14$. The signaling pathways for branching inhibition of the tested compounds were thus confirmed to be SL signaling pathways, rather than toxicity. Examining the effects of compounds 5 and 6 on the shoot branching of $d 10$ and $d 14$ mutants revealed that the $d 14$ mutant exhibited a phenotype different from the d10 mutant. After 20 days of compound treatment, the tiller number of the $d 14$ mutant was not suppressed (Fig. 4). Compounds 5 and 6 significantly inhibited branching in $d 10$ but not in $d 14$. This preliminarily proved that the signaling pathways of these compounds regulating branching were SL signaling pathways.

\section{Effects of Chemicals on the D14-d53 Interaction}

As mentioned above, we found that compounds 5 and 6 were ineffective for inhibiting shoot branching in rice $d 14$ mutants. One possible explanation is that compounds 5 and 6 are recognized in the SL signaling pathway. In previous reports, the yeast two-hybrid $(\mathrm{Y} 2 \mathrm{H})$ system has been used to examine the SLdependent interactions between D14 and D53 or d53 (Zhou et al. 2013). To ascertain whether compounds that inhibit shoot branching can induce interactions between the SL receptor D14 and the coreceptor d53, we performed $\mathrm{Y} 2 \mathrm{H}$ experiments for compounds 5, 6, and 18, revealing that D14-D53 interactions occurred in all three, similar to GR24 (Fig. 5). These results were consistent with our bioassay results. Various moieties of compounds 5 and 6 were found to be linked to the D-ring via ether, and compound 18 was a carboxylic acid compound in which the moieties were linked via ester bonds. By conducting further $\mathrm{Y} 2 \mathrm{H}$ experiments, we also found that GR24 induced D14-d53 interactions at a concentration of $5 \mu \mathrm{M}$, whereas compounds 5,6 , and 18 induced them at a concentration of $50 \mu \mathrm{M}$. These results indicated that these compounds inhibited shoot branching activity by inducing the formation of the SL receptor complex, but not as strongly as GR24. These data conclusively demonstrated that these compounds inhibited shoot branching by inducing receptor complex formation between D14 and D53.

\section{Effects of chemicals on the expressions of SL-related genes}


As SLs can upregulate the expressions of OsTB1, OsD53, and OsCKX9, we explored whether these genes were regulated upon treatment with our studied compounds. Examining the expression levels of these four genes in the leaf and shoot bases of rice seedlings treated with compound 18 revealed that expressions of $O s T B 1, O s D 53$, and $O s C K X 9$ were strongly induced by compound 18 in the leaves after $4 \mathrm{~h}$ and slightly increased in shoot bases after $8 \mathrm{~h}$ (Fig. 6). These results suggested that most of the changes at the transcript level were the same as those observed upon GR24 treatment. This molecular assay could also be used to explain the signaling pathways of compounds, similar to the $\mathrm{Y} 2 \mathrm{H}$ experiments. This indicates that compound 18 participates in the SL pathway.

\section{Discussion}

To date, few SL analogs have been used for tillering inhibition, not only because the hormonal functions of SLs were only recently discovered but also because of their high synthesis costs. Here, we economically synthesized 29 compounds using a minimalistic synthesis method and tested their tillering inhibition activities. We found that some of these compounds could significantly inhibit the outgrowth of branches. Furthermore, $\mathrm{Y} 2 \mathrm{H}$ experiments and transcriptional analysis showed that these compounds exerted their activity through the SL signaling pathway.

\section{Conserved Domains of Compounds for Repressing Branching}

The structure-activity relationships and signal triggering mechanisms of SLs have not yet been revealed. A study into the structure-activity relationships of SLs underlined that the D-ring is a conserved molecular domain (Zhao et al. 2015); it has been demonstrated as an important part not only in an SL bioassay based on axillary bud length after direct SL application but also through the mechanism of molecular SL recognition. In a previous study, rice D14 hydrolyzed SLs to produce D-OH and D14, then formed a CLIM with D-OH. Although the structural plasticities of D3 and D14 are still being investigated in SL signaling, it has been established that the methyl substituent in the D-ring cannot form covalent bonds with D14 (Yao et al. 2016). In addition, as a germination stimulant, the methyl substituent in the D-ring is essential for bioactivity (Boyer et al. 2012). A demethyl analog (chlorpyrifos; CPF) was reported in a previous study (Fukui et al. 2013). In our assay, we found that CPF was inactive in inhibiting the shoot branching of the $d 10$ mutant, but its concentration was only $1 \mu \mathrm{M}$. We also found that compounds lacking 4 'methyl could only inhibit tillering at higher concentrations. In our study, the methyl substituent in the D-ring was removed; we found that some compounds could inhibit the tillering number, although the application concentration required was higher than that of GR24. We also confirmed that methyl substituents in the D-ring are not necessary for SL signaling. However, the structure-activity relationships of SLs are different for different species and different biological functions.

\section{Non-conserved Domains of Compounds for Repressing Branching}


The ABC ring is considered to be a non-conserved domain of SLs (Boyer et al. 2012; Fukui et al. 2011b), but to date, no definitive conclusion has been reached regarding its structural requirements. Here, we designed the $\mathrm{ABC}$ rings of compounds into different structures; we replaced the enol ether bond with an ether or ester bond. We found that some phenols, carboxylic acids, and esters were effective in inhibiting the tillering number of $d 10$ mutants. Compounds with different structures may guide different signaling pathways because some of these compounds also affect root length and plant height. While we summarized the essential requirements of the $A B C$ ring in 29 compounds, we did not obtain the exact structural requirements. By analyzing the types of effective compounds, we found that phenols were generally more effective than carboxylic acids. Compound degradation is closely related to receptor binding and signal transduction. In addition, we found that compounds showed different levels of effectiveness in the same species. These observations suggest that broken chemical bonds are also very important in signal transmission and the structural requirements of the $A B C$ ring warrant further investigation.

\section{Future Analogs}

Through our study of 29 compounds, we investigated the structure-activity relationships of SLs and provided a direction for the further development of analog structures. The high synthesis costs of SLS has always been the biggest problem. Removing the methyl substituent in the D-ring greatly reduces the synthesis costs of SLs and simplifies the required synthesis steps. Replacing the enol ether bond with an ether bond may also be another important idea for analog structures. The structure of the ABC ring is still unknown, but we speculate that it is related to the signaling of different hormones. D14 has been reported to form a complex with D-ring and interact with the rice DELLA protein, the key regulator of gibberellin (GA) signaling (Nakamura et al. 2013). Therefore, the different structures of the ABC ring may affect the interactions between D14 and different proteins. In addition, the introduction of heteroatoms to the ABC ring can also change the solubility of compounds, for example, compound 14 , which is water-soluble; this approach will likely be more widely used in the future.

\section{Conclusion}

Here, our investigation of structure-activity relationships demonstrated that the methyl substituent in the D-ring is not an essential component of compounds that inhibit tiller bud outgrowth. In addition, our results indicate that the differences in chemical bond connections between $\mathrm{D}$ - and $\mathrm{C}$-ring greatly influence branching inhibition. Furthermore, our results from $d 14$ mutants and $\mathrm{Y} 2 \mathrm{H}$ experiments provide evidence to explain the biological activity of SL regarding the tested chemicals. As the structures of D14 and D3 proteins have been clarified, several mechanisms for SL signaling have been developed. These findings are instructive for understanding the structure-activity relationships of SLs with respect to shoot branching inhibition, and revealing the molecular recognition mechanisms of SLs.

\section{Declarations}


Sequence data from this article can be found in Rice Annotation Project database under the following accession numbers: Actin (Os03g0718100), D10 (Os01g0746400), D14

(Os03g0203200), D53 (Os11g0104300), OsCKX9 (Os05g0374200), and OsTB1(Os03g0706500).

\section{Funding}

This work was supported by Jiangsu Agriculture Science and Technology Innovation Fund (Grant number CX (20) 3175).

\section{Disclosures}

The authors have no conflicts of interest to declare.

\section{Acknowledgments}

We thank Dr. Junko Kyozuka for the gift of $d 10$ and $d 14$ mutant seeds.

\section{Author Contributions}

C.D., L.W., and Y.D. designed the research. S.W. synthesized the compound. Y.L., and C.R. performed the experiments. C.D. C.R., and Y.L. analyzed the data and prepared the manuscript.

\section{References}

1. Akiyama K, Hayashi H (2006) Strigolactones: chemical signals for fungal symbionts and parasitic weeds in plant roots. Ann Bot 97:925-931

2. Al-Babili S, Bouwmeester HJ (2015) Strigolactones, a novel carotenoid-derived plant hormone. Annu Rev Plant Biol 66:161-186

3. Alder A, Jamil M, Marzorati M, Bruno M, Vermathen M, Bigler $P$ et al (2012) The path from $\beta$-carotene to carlactone, a strigolactone-like plant hormone. Science 335:1348-1351

4. Arite T, Iwata $\mathrm{H}$, Ohshima K, Maekawa M, Nakajima M, Kojima M et al (2007) DWARF10, an RMS1/MAX4/DAD1 ortholog, controls lateral bud outgrowth in rice. Plant J 51:1019-1029

5. Arite T, Umehara M, Ishikawa S, Hanada A, Maekawa M, Yamaguchi S et al (2009) d14, a strigolactone-insensitive mutant of Rice, shows an accelerated outgrowth of tillers. Plant Cell Physiol 50:1416-1424

6. Boyer FD, de Saint Germain A, Pillot JP, Pouvreau JB, Chen VX, Ramos S et al (2012) Structureactivity relationship studies of strigolactone-related molecules for branching inhibition in garden pea: molecule design for shoot branching. Plant Physiol 159:1524-1544

7. Cook CE, Whichard LP, Turner B, Wall ME, Egley GH (1966) Germination of witchweed (Striga lutea Lour.): isolation and properties of a potent stimulant. Science 154:1189-1190

8. Duan J, Yu H, Yuan K, Liao Z, Meng X, Jing Y et al (2019) Strigolactone promotes cytokinin degradation through transcriptional activation of CYTOKININ OXIDASE/ DEHYDROGENASE 9 in rice. 
Proc Natl Acad Sci U S A 116:14319-14324

9. Dun EA, de Saint Germain A, Rameau C, Beveridge CA (2012) Antagonistic action of strigolactone and cytokinin in bud outgrowth control. Plant Physiol 158:487-498

10. Fukui K, Ito S, Asami T (2013) Selective mimics of strigolactone actions and their potential use for controlling damage caused by root parasitic weeds. Mol Plant 6:88-99

11. Fukui K, Ito S, Ueno K, Yamaguchi S, Kyozuka J, Asami T (2011) New branching inhibitors and their potential as strigolactone mimics in rice. Bioorg Med Chem Lett 21:4905-4908

12. Gomez-Roldan V, Fermas S, Brewer PB, Puech-Pages V, Dun EA, Pillot JP et al (2008) Strigolactone inhibition of shoot branching. Nature 455:189-194

13. Jiang L, Liu X, Xiong G, Liu H, Chen F, Wang L et al (2013) DWARF 53 acts as a repressor of strigolactone signalling in rice. Nature 504:401-405

14. Koltai H (2011) Strigolactones are regulators of root development. New Phytol 190:545-549

15. Mach J (2015) Strigolactones regulate plant growth in Arabidopsis via degradation of the DWARF53Like Proteins SMXL6, 7, and 8. Plant Cell 27:3022-3023

16. Mangnus EM, Dommerholt FJ, Dejong RLP, Zwanenburg B (1992) Improved synthesis of strigol analogue GR24 and evaluation of the biological activity of its diastereomers. J Agric Food Chem 40:1230-1235

17. Mangnus EM, Zwanenburg B (1992) Tentative molecular mechanism for germination stimulation of Striga and Orobanche seeds by strigol and its synthetic analogues. J Agric Food Chem 40:10661070

18. Nakamura H, Xue YL, Miyakawa T, Hou F, Qin HM, Fukui K et al (2013) Molecular mechanism of strigolactone perception by DWARF14. Nat Commun 4:2613

19. Scaffidi A, Waters MT, Ghisalberti EL, Dixon KW, Flematti GR, Smith SM (2013) Carlactoneindependent seedling morphogenesis in Arabidopsis. Plant J 76:1-9

20. Scaffidi A, Waters MT, Sun YMK, Skelton BW, Dixon KW, Ghisalberti EL et al (2014) Strigolactone hormones and their stereoisomers signal through two related receptor proteins to induce different physiological responses in Arabidopsis. Plant Physiol 165:1221-1232

21. Seto Y, Yasui R, Kameoka H, Tamiru M, Cao M, Terauchi R et al (2019) Strigolactone perception and deactivation by a hydrolase receptor DWARF14. Nat Commun 10:191

22. Umehara M, Cao MM, Akiyama K, Akatsu T, Seto Y, Hanada A et al (2015) Structural requirements of strigolactones for shoot branching inhibition in Rice and Arabidopsis. Plant Cell Physiol 56:10591072

23. Umehara M, Hanada A, Yoshida S, Akiyama K, Arite T, Takeda-Kamiya N et al (2008) Inhibition of shoot branching by new terpenoid plant hormones. Nature 455:195-200

24. Wang B, Smith SM, Li J (2018) Genetic regulation of shoot architecture. Annu Rev Plant Biol 69:437468 
25. Wang L, Wang B, Jiang L, Liu X, Li X, Lu Z et al (2015) Strigolactone signaling in Arabidopsis regulates shoot development by targeting D53-Like SMXL repressor proteins for ubiquitination and degradation. Plant Cell 27:3128-3142

26. Xie X (2016) Structural diversity of strigolactones and their distribution in the plant kingdom. J Pestic Sci 41:175-180

27. Yao J, Scaffidi A, Meng Y, Melville KT, Komatsu A, Khosla A, Nelson et al (2021) Desmethyl butenolides are optimal ligands for karrikin receptor proteins. New Phytol 230:1003-1016

28. Yao R, Ming Z, Yan L, Li S, Wang F, Ma S et al (2016) DWARF14 is a non-canonical hormone receptor for strigolactone. Nature 536:469-473

29. Zhang Y, van Dijk AD, Scaffidi A, Flematti GR, Hofmann M, Charnikhova T et al (2014) Rice cytochrome P450 MAX1 homologs catalyze distinct steps in strigolactone biosynthesis. Nat Chem Biol 10:1028-1033

30. Zhao LH, Zhou XE, Yi W, Wu Z, Liu Y, Kang Y et al (2015) Destabilization of strigolactone receptor DWARF14 by binding of ligand and E3-ligase signaling effector DWARF3. Cell Res 25:1219-1236

31. Zhou F, Lin Q, Zhu L, Ren Y, Zhou K, Shabek N et al (2013) D14-SCF ${ }^{\mathrm{D} 3}$-dependent degradation of D53 regulates strigolactone signalling. Nature 504:406-410

32. Zwanenburg B, Mwakaboko AS, Reizelman A, Anilkumar G, Sethumadhavan D (2009) Structure and function of natural and synthetic signalling molecules in parasitic weed germination. Pest Manag Sci 65:478-491

\section{Figures}

\section{Figure 1}

Classification and synthesis steps for compounds.

(a) A two-step synthesis route for compounds used in this study. (b) Phenolic compounds (1-14). (c) Carboxylic acid compounds (15-26). (d) Ester compounds (27-28). (e) Amino acid-derived compound (29).

\section{Figure 2}

The phenotype of the $d 10$ mutant after treatment with each of the 29 compounds.

(a) Plant phenotypes treated with phenolic compounds. (b) Plant phenotypes treated with carboxylic acid compounds. (c) Plant phenotypes treated with ester compounds. (d) Plant phenotypes treated with amino 
acid-derived compounds (scale bar $=5 \mathrm{~cm}$ ). (e) Tiller number, plant height, and root length of the $d 10$ mutant after 20 days of treatment with the compound or dimethyl sulfoxide (DMSO). Data are presented as the mean \pm standard deviation ( $S D ; n=6)$. Fold changes were used for heatmaps. Values are equal to the tiller number of the compound-treated sample divided by the tiller number of the control. $P$ values represent significant differences between compound and DMSO treatments: ${ }^{P}<0.05, * * P<0.01$, and *** $P<0.001$, as determined by student's $t$-tests.

\section{Figure 3}

Effects of compound concentration and applied organizations on tillering inhibition of rice.

(a) Tiller number ratio between compound- and DMSO-treated samples at different concentrations. (b) Tiller number ratio between compound- and DMSO-treated samples. Tiller number is after 20 days of treatment with the compound or DMSO.

\section{Figure 4}

Effects of compounds on $d 10$ and $d 14$ mutants.

(a) Phenotypes of $d 10$ and $d 14$ mutants after compound treatment (scale bar $=5 \mathrm{~cm}$ ). (b) Tiller number ratio between compound- and DMSO-treated samples. Data are presented as the mean \pm standard error $(S E ; n=6)$. $P$ values represent significant differences between treatment and DMSO treatments: ${ }^{*} P$ $<0.01$, as determined by student's $t$-tests.

\section{Figure 5}

Promotion of d53-D14 interactions by compounds.

$\mathrm{Y} 2 \mathrm{H}$, showing that $\mathrm{d} 53$ interacts with $\mathrm{D} 14$ in the presence of compounds and GR24. Yeast transformants were spotted on the control medium (SD-Leu/-Trp): - LT and selective medium (SD-Leu/-Trp/-His/Ade): -LTHA. AD, pGADT7; BD, pGBKT7.

\section{Figure 6}

Effect of compound $18(50 \mathrm{mM})$ on transcript levels of strigolactone-downstream response genes in leaf and shoot bases. Plants were treated by application through the hydroponic fluid. Data are presented as 
the mean $\pm S E(n=3)$. P values represent significant differences between compound and DMSO treatments: ${ }^{*} \mathrm{P}<0.05$, ${ }^{\star *} \mathrm{P}<0.01$, and ${ }^{\star \star \star} \mathrm{P}<0.001$, as determined using student's $t$-tests. 